wristband the nurse scans the barcode on the medicine labels of cytostatic drugs. If the dose being scanned corresponds to a pharmacistapproved medicines order and the patient is due for this dose, administration is automatically documented. However, if the dose does not correspond to a valid order, the application issues a warning. Every action performed with PDAs is recorded in the database.

Results During the first year since its introduction, this system has been used in 709 oncology-haematological and rheumatologic patients (24.8\% haematology, $49.1 \%$ oncology, $22.6 \%$ rheumatology patients), 3995 medicine orders have been scanned (22.2\% haematology, $60.2 \%$ oncology, $17.6 \%$ rheumatology) and 11435 doses identified (12.3\% haematology, $80.8 \%$ oncology, $6.9 \%$ rheumatology).

$99.7 \%$ of the doses identified with this system were administered while the remaining $0.3 \%$ were not administered to patients due to the occurrence of several adverse reactions.

Variables validated by the scan were: patient, drug administration sequence, start and end times. Possible errors detected: incorrect order of administration, drug already administered and drug selected that does not belong to the scanned patient. During the study period we detected 2 cases of selected drug that did not belongs to scanned patient. The system issued a warning that prevented the wrong drug being administered to the patient, probably the worst error with cytostatic drugs administration.

Conclusions The implementation of barcode medicines verification technology embedded in an eMAR in a day hospital acted as an additional safety net in medicines administration and patient safety. This system also improved treatment efficiency and achieved greater interdisciplinary collaboration.

No conflict of interest.

\section{GRP-032 BENEFICIAL EFFECT OF HOSPITAL PHARMACIST PARTICIPATION IN INTENSIVE CARE ROUNDS: REDUCTION IN MEDICINES ERRORS AND HOSPITAL COSTS}

doi:10.1136/ejhpharm-2013-000276.032

${ }^{1} \mathrm{AL}$ de Goede, 'PMLA van den Bemt, 'ML Becker, ${ }^{2} \mathrm{~J}$ van Bommel, ${ }^{3} \mathrm{NGM}$ Hunfeld. 'Erasmus MC, Department of Hospital Pharmacy, Rotterdam, The Netherlands; 2Erasmus MC, Department of Intensive Care, Rotterdam, The Netherlands; ${ }^{3}$ Erasmus MC, Department of Hospital Pharmacy and Department of Intensive Care, Rotterdam, The Netherlands

Background Medicines errors may result in patient harm. Especially in intensive care patients, adverse drug events caused by medicines errors are common. Interventions by hospital pharmacists have been shown to reduce adverse drug events and costs in intensive care units (ICUs).

Purpose To evaluate the effect of active participation of a hospital pharmacist in the ICU on medicines errors and hospital costs.

Materials and Methods A three-month pilot study was performed at the adult 32-bed ICU of the academic hospital Erasmus MC. Four hospital pharmacists were trained in specific aspects and protocols of intensive care. From July to September 2011, each patient's medicines profile was reviewed weekly using a standardised written form and a pharmacist was present on rounds. Potential medicines errors requiring intervention were documented and discussed during the round. In addition, the amount of time spent performing clinical activities at the ICU was recorded.

Results 267 medicines reviews were performed for a total of 169 patients in 51 rounds. 288 interventions for a total of 120 drugs were made. About $60 \%$ of the medicines reviews resulted in at least one intervention with an acceptance rate of $56 \%$. Non-acceptance was mainly due to a lack of information at the time the medicines review was performed. $30 \%$ of interventions were relating to unnecessary drug use, $24 \%$ to drug omission and $17 \%$ to a wrong dose. Time spent on medicines reviews and visiting rounds was 7.3 hour per week. Based on these results we developed a business case for structural participation of a hospital pharmacist at the ICU.

Conclusions Participation of a hospital pharmacist in ICU rounds improves medicines safety and can be cost-effective. The pilot study and business case have resulted in the appointment of $0.5 \mathrm{FTE}$ hospital pharmacist in the ICU.

No conflict of interest.

\section{GRP-033 BENZODIAZEPINE DRUG ABUSE AMONG INTRAVENOUS DRUG USERS}

doi:10.1136/ejhpharm-2013-000276.033

II Bacovich, ${ }^{2} \mathrm{~J}$ Delás, ${ }^{3} \mathrm{~N}$ El Hilali, ${ }^{3}$ Javier, ${ }^{3} \mathrm{M}$ Aguas, 'V González, 'R Kistmacher, 'O Díaz, 'L Andreo, 'J Camí. 'SAPS, Creu Roja, Barcelona, Spain; ${ }^{2 H O S P I T A L ~ S A G R A T ~}$ COR, Internal Medicine, Barcelona, Spain; ${ }^{3}$ HOSPITAL SAGRAT COR, Pharmacy, Barcelona, Spain

Background Benzodiazepine drug abuse is frequent in the general population. The reasons for this could be very diverse.

Purpose To review the role of benzodiazepine in intravenous drug users.

To find out which benzodiazepines are most used in this group and sought after on the black market.

Materials and Methods We interviewed five intravenous drug users of heroin or cocaine in Barcelona about their associated use of benzodiazepine. They were trained to interview other intravenous drug users with the same questionnaire that they had answered. All of them had looked for benzodiazepines on the illegal market at least once.

Results The analysis of the first 25 questionnaires answered showed that the most used benzodiazepine was clonazepam, used by $72 \%$ and the drugs used differed in half life and effects.

Conclusions Benzodiazepines selected by this sample of patients did not meet criteria for half-life or the main indications. They may simply be a reflection of which benzodiazepines are most prescribed nowadays by psychiatrists in the community.

\section{Abstract GRP-033 Table 1}

\begin{tabular}{lll}
\hline & N: $\mathbf{2 5}$ & \% \\
\hline Clonazepam & 18 & 72 \\
Alprazolam & 17 & 68 \\
Clorazepate dipotassium & 5 & 20 \\
Lorazepam & 4 & 16 \\
Diazepam & 4 & 16 \\
Midazolam & 2 & 8 \\
Lormetazepam & 2 & 8 \\
Zolpidem & 1 & 4 \\
\hline
\end{tabular}

No conflict of interest.

\section{GRP-034 BLOOD PRESSURE CONTROL AND ANTIHYPERTENSIVE PHARMACOTHERAPY PATTERNS IN A HYPERTENSIVE PORTUGUESE POPULATION}

doi:10.1136/ejhpharm-2013-000276.034

'M Morgado, ${ }^{2} \mathrm{~J}$ Soares, ${ }^{2} \mathrm{~A}$ Almeida. ${ }^{1}$ Hospital Centre of Cova da Beira, Pharmaceutical Services, Covilhã, Portugal; 'University of Beira Interior, Health Sciences Faculty, Covilhã, Portugal

Background Interventions to improve blood pressure (BP) control in hypertension have had limited success in clinical practise despite evidence of cardiovascular disease prevention in randomised controlled trials.

Purpose To evaluate BP control and patterns of antihypertensive pharmacotherapy in a population in the Central Region of Portugal, attending a hospital outpatient clinic for routine follow-up. 